Case Reports

\title{
Non-Specific Interstitial Pneumonia Overlapping Tuberculosis: A Diagnostic Challenge in Elderly
}

\author{
Pradeep Chakaravarthy, Divanshee Sharma, Manjunath Totaganti, Jithesh G, Mukesh Bairwa and Ravikant \\ Department of Internal Medicine, All India Institute of Medical Sciences, Rishikesh, India
}

\author{
Article history \\ Received: 28-12-2020 \\ Revised: 06-04-2021 \\ Accepted: 09-04-2021 \\ Corresponding Author: \\ Ravikant \\ Department of Internal \\ Medicine, All India Institute of \\ Medical Sciences, Rishikesh, \\ India \\ Email: drkantr2006@gmail.com
}

\begin{abstract}
Non-Specific Interstitial Pneumonia (NSIP) is linked to numerous etiologies, including connective tissue diseases, certain drugs, HIV and idiopathic causes. We present a case of an 82-year-old woman with subacute onset shortness of breath as the presenting feature of Non-specific interstitial pneumonia. Chest imaging demonstrated bilateral infiltrates and tentatively diagnosed as NSIP and started on Tyrosine kinase inhibitor - Nintedanib. But she worsened symptomatically and on further evaluation, Mycobacterium tuberculosis had been detected in her Sputum. Anti-tuberculous therapy and Tyrosine Kinase Inhibitor (TKI) resolved her pulmonary symptoms to an extent and she was discharged on Long term oxygen therapy. In this case, we encountered a Tuberculosis (TB) case with NSIP where NSIP could overlap the diagnosis of TB and we should have a strong suspicion of TB in geriatric people.
\end{abstract}

Keywords: Non-Specific Interstitial Pneumonia, Tuberculosis, Rheumatoid Arthritis, Osteoarthritis, Steroids, Geriatric Population

\section{Introduction}

An 82-year-old female resident of a hilly area, homemaker, non-smoker/alcoholic, a known hypertensive for eight years non-compliance to antihypertensive therapy and had drug history of steroids (prednisolone $10 \mathrm{mg}$ ) for three years for seronegative arthritis prescribed by local physician presented to Emergency Department with complaints of insidious onset and gradually progressive shortness of breath for four months (initially MMRC-II now progressed to MMRC-IV) and mild cough with whitish expectoration for last one month and also had a history of generalized weakness. No history of fever, headache, weight loss, haemoptysis, abdominal pain, burning micturition, decreased urine output, night sweats and chest pain.

In ED, she was slightly tachypneic with a respiratory rate of 28 , a saturation of $75 \%$ on the right index finger, mild tachycardia with 103 beats per minute and afebrile. General examination was unremarkable except for joint deformity in the 4th distal interphalangeal joint on the right hand and started on oxygen supplementation with face mask at 6 litres per minute and her Arterial blood gas analysis revealed moderate hypoxemia with $\mathrm{PaO}_{2} / \mathrm{FiO}_{2}$ ratio of 140 . Her baseline investigations revealed a prerenal pattern of Acute kidney injury with other parameters within normal limits as shown in Table 1. Electrocardiogram revealed features suggestive of she has left ventricular hypertrophy. Bedside chest x-ray revealed patchy consolidation over right lower lobe and extended reticular opacities over middle and lower lobe with increasing intensity in craniocaudal direction as shown in Fig. 1 and empirically started on antibiotics - ceftriaxone and Azithromycin intravenously. She had been screened for Corona by RT-PCR and Contrast-Enhanced Computed Tomography (CECT) was done and in imaging, she had features suggestive of Interstitial Lung Disease which is likely of Fibrotic Non-Specific Interstitial Pneumonia pattern with few sub-centimetric and enlarged lymph nodes were seen in perivascular, bilateral lower parabrachial and subcarinal areas with few calcifications. She had no history compatible with any connective tissue diseases and all serum rheumatologic markers were negative.

She was thoroughly examined after her RT-PCR for COVID was found negative. Clinically she had bilateral crepitation over bilateral basal zones and bronchial breath sound over the right basal zone. Pulmonary Function Test could not be done due to COVID pandemic. Initially, she was managed on the lines of NSIP and TKI- Nintedanib was started at a dose of $150 \mathrm{mg}$ BD. Hypothetically her symptoms worsened. Hence, Sputum was sent for analysis of Gram-staining, Acid-fast bacilli and CBNAAT (Cartridge-Based Nucleic Acid Amplification Test) for Mycobacterium, in which nucleic acid of Mycobacterium tuberculosis was detected in CBNAAT. She was started on Anti-tuberculous drugs. She was evaluated for her history of joint pain, which was suggestive of Osteoarthritis (fig - X-ray hand). During the course of treatment, her oxygen requirement decreased gradually and her sputum production also decreased. But she had significant exertional dyspnea and she needs 
minimal oxygen requirement at rest and has been discharged with Long term oxygen therapy.

\section{Discussion}

Tuberculosis (TB) is one of the most notorious and most lethal infectious diseases globally and mostly in developing countries like India. It is estimated that one-third of the world's population is infected with the tubercle Bacillus-Mycobacterium Tuberculosis (Mtb) and around 7 to 8 million people develop the disease each year (7). The elderly population accounts of all ethnic groups represent the majority of the reservoir of infection and clinical features in older adults may be atypical, nonspecific and confused with concomitant age-related diseases. With ageing comes the major comorbidities due to many factors and underlying acute or chronic illnesses, malnutrition and biological changes, which can disrupt integument barriers, impair microbial clearance mechanisms and contribute to the expected age-associated decline in cellular immune responses infecting agents such as Mtb. This causes a lot of confusion in the diagnosis of TB in the elderly and unfortunately, many older adults being succumbed to TB (Rajagopalan and Yoshikawa, 2000).

In a study by Chung et al. (2004) 143 consecutive patients with Interstitial Pulmonary Fibrosis (IPF) were analyzed and found five times more chance of TB incidence than the general population.

Interstitial Lung Diseases are a group of diffuse infiltrative lung disorders characterized by the presence of inflammation and altered lung interstitial. Among ILD, Non-Specific Interstitial Pneumonia (NSIP) can be idiopathic or associated with connective tissue disease, HIV infection, toxins, or numerous other causes. Various causes of NSIP include:

- Connective Tissue Disease (CTD): NSIP is a typical pattern in most CTD's like systemic sclerosis, polymyositis/dermatomyositis, rheumatoid arthritis and Sjogren syndrome

- Drug-induced: Amiodarone, methotrexate, nitrofurantoin, chemotherapeutic agents and statin therapy are the drugs that can cause fibrosis and the usual pattern is NSIP (Schwaiblmair et al., 2012)

- HIV infection: Incidence of NSIP in HIV became less common after the introduction of retroviral therapy

- Some patients presenting with Interstitial pneumonia have NSIP patterns in Biopsy and some autoimmune features that don't match any specific CTD's

- Hypersensitivity pneumonitis patients sometimes lack granuloma and multinucleated cells in histology and will have an appearance of NSIP

- Other diseases such as IgG4-related systemic disease, familial interstitial pneumonia and graft versus host disease also have NSIP pattern
NSIP is a rare entity and comprises 14 to $36 \%$ of Idiopathic Pulmonary Fibrosis cases (IPF). Among IPF, Usual Interstitial Pneumonia (UIP) (50 to 60\%) is more common than NSIP (14-36\%), desquamative interstitial pneumonia, respiratory bronchiolitis-associated interstitial lung disease (DIP/RB-ILD) (10-17\%) and acute interstitial pneumonia (AIP) (0 to $2 \%)$. Idiopathic NSIP is more common in middle-aged females who are non-smokers and NSIP due to CTD's has an equal incidence in men and women (Thomas et al., 2002).

NSIP is mostly suspected by the findings in HighResolution Computed Tomography(HRCT) of the thorax and in 2008, the ATS expert consensus described the radiological features and biopsy findings. The abnormalities in HRCT thorax are predominantly lower lung involvement $(92 \%)$ and were either diffuse $(47 \%)$ or peripheral $(46 \%)$ in the axial dimension. Common findings noted were reticular abnormalities (87\%), traction bronchiectasis (82\%) and lobar volume loss (77\%), but ground-glass attenuation (44\%), subpleural sparing $(21 \%)$ and per bronchial thickening $(6.6 \%)$ were also seen. Subpleural sparing, when present, suggests NSIP but is not pathognomonic (Lee et al., 2019).

Our case had typical clinical and radiological features for NSIP like subacute, progressive dyspnea and absence of clubbing at presentation. Also, the High-Resolution Computed Tomography (HRCT) features of our case has typical radiological features of fibrotic NSIP, as shown in the Fig. 3:

- Ground glass opacities

- Traction bronchiectasis

- Bilateral basal zone involvement

- Inter/intralobular septal thickening and

○ No evidence of honeycombing

Comparatively, the mortality in the elderly due to TB is six times higher than the younger people, which denotes a significant difference and this may be due to the physicians missing the diagnosis of TB in the elderly. TB, a preventable and treatable disease, needs increased awareness in early recognition and better medical and supportive care among older adults.

She also had lobar consolidation, cysts, nodules and areas of low attenuation, which is not a typical feature of NSIP (Anirudhan et al., 2018). It developed a suspicion of another pathology and had been evaluated further, which led to the diagnosis of Pulmonary Tuberculosis.

Even though she was on steroids for her arthritis, which increases the risk of reactivation/new infection of $\mathrm{Mtb}$, she was completely evaluated. Her serological markers for connective tissue disease like RA, ANA and anti-CCP were negative. Clinically, there was a deformed joint over the distal interphalangeal joint on the 4th finger (Fig. 2). Her age, clinical history, investigations and imaging point towards Osteoarthritis, which was mismanaged with steroids. 
Steroids play an essential role in reactivation of latent TB and even modify the typical presentation of TB, paving the way for atypical presentations and may increase the incidence of extra pulmonary TB and it also had a significant role in the treatment of many forms of extra pulmonary TB (Cisneros and Murray, 1996).

In our case, she had a history of steroids, which may pose as a risk factor for TB. Still, she had very minimal features suggestive of Tuberculosis like generalized weakness and patchy consolidation in the lower lobe, which was not usually seen in Pulmonary Tuberculosis, Sputum had been sent and mycobacterium tuberculosis had been detected. Hence, in the case of the elderly, imaging is not the confirmed diagnostic criteria and should be thoroughly investigated based on clinical history, examination findings and response to treatment.

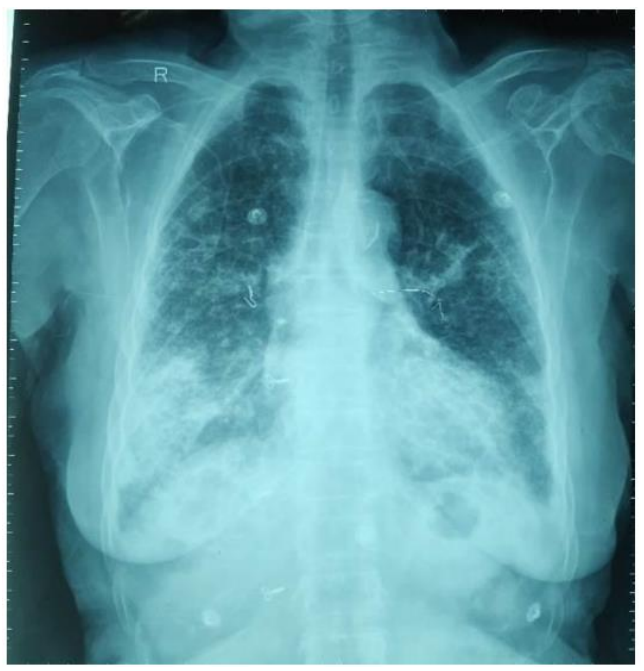

Fig. 1: chest x-ray- PA view showing bilateral reticulonodular opacities more predominantly involving basal zones and patchy consolidation on right lower lobe

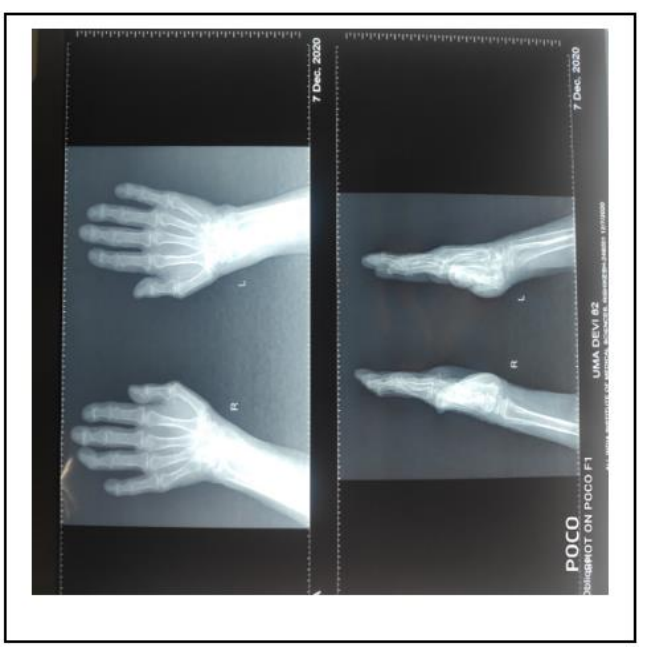

Fig. 2: X-RAY Both hands showing dysarthosis over 4th digit suggestive of Osteoarthritis

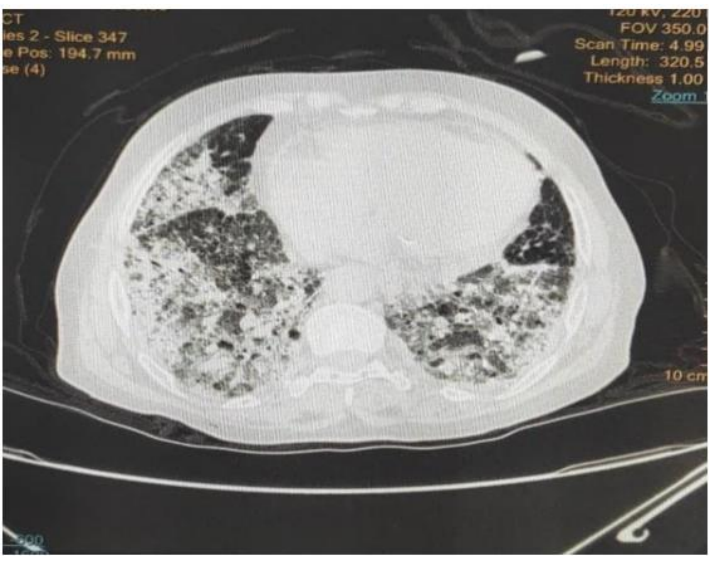

Fig. 3: High-Resolution Computed Tomography (HRCT) images in Nonspecific Interstitial Pneumonia (NSIP). HRCT through the lower lungs in a patient with NSIP shows confluent ground-glass/reticular abnormality and mild traction bronchiectasis

Table 1: BASELINE INVESTIGATIONS

\begin{tabular}{lllll}
\hline DATE & $\mathbf{0 4 / 1 2}$ & $\mathbf{0 9 / 1 2}$ & $\mathbf{1 4 / 1 2}$ & $\mathbf{1 7 / 1 2}$ \\
\hline HB & 14.93 & 12.96 & 13.58 & \\
TLC & 8700 & 7300 & 7600 & \\
DLC & $87 / 5.5 / 6.5$ & $63 / 15 / 9.6$ & $68 / 14 / 7$ & \\
Platelet count & 131 & 139 & 186 & \\
Blood Urea & 51 & 76 & 21 & \\
S. Creatinine & 1.10 & 1.05 & 0.82 & \\
S. Na+ & 139 & 140.1 & 142 & \\
S. K+ & 5 & 3.8 & 4 & \\
S. Calcium & 8.5 & 8.44 & & \\
S. uric acid & 8.7 & 6.79 & & \\
Phosphorus & 3.8 & 4 & & \\
PT/INR & $13.5 / 1$ & & & \\
T.BIL & 0.57 & & & 0.55 \\
D.BIL & .28 & & & 0.31 \\
SGPT & 14.2 & & & 17.4 \\
SGOT & 24.2 & & & 21.1 \\
ALP & 224.2 & & & 187 \\
GGT & 12 & & & 6.7 \\
T. Protein & 6.8 & & & 4.33 \\
S. Albumin & 3.9 & & &
\end{tabular}

\section{Conclusion}

Tuberculosis in the elderly does not follow the predictable course. We can say in this age group; it's all together with a different disease. Interstitial lung diseases are the disorders that are being frequently diagnosed nowadays due to the increased use of HRCT after the Coved pandemic and hence knowledge about these disorders are essential. Thorough knowledge about these disorders should be provided to all physicians, especially with a high prevalence of TB. There would not be any delay and mismanagement among older adults where atypical presentations of these diseases were common. Hence, we presented an interesting case of Tuberculosis 
and NSIP- a rare phenomenon that may present as a diagnostic challenge. Clinicians involved in the management of ILD should take into consideration of possible concomitant infection.

\section{Acknowledgement}

We would like to thank this patient for her cooperation and assistance for giving us a chance to improve our knowledge.

\section{Author's Contributions}

Pradeep Chakaravarthy: Manuscript preparation, data collection and case reporting.

Divanshee Sharma: Manuscript preparation, literature search and editing.

Manjunath Totaganti: Data collection and editing.

Jithesh G: manuscript review.

Mukesh Bairwa and Ravikant: Manuscript review and guidance.

\section{Ethics}

Written informed consent was taken from the patient.

\section{References}

Anirudhan, V. K., Prasannan, A., \& Devadas, S. (2018). Non Specific Interstitial Pneumonia: A Case Report. Journal of Clinical \& Diagnostic Research, 12(1).

Cisneros, J. R., \& Murray, K. M. (1996). Corticosteroids in tuberculosis. Annals of Pharmacotherapy, 30(11), 1298-1303. doi.org/10.1177/106002809603001115
Lee, J., Kim, Y. H., Kang, J. Y., Jegal, Y., \& Park, S. Y. (2019). Korean guidelines for diagnosis and management of interstitial lung diseases: Part 3. Idiopathic nonspecific interstitial pneumonia. Tuberculosis and respiratory diseases, 82(4), 277-284. doi.org/10.4046/trd.2018.0092

Rajagopalan, S., \& Yoshikawa, T. T. (2000). Tuberculosis in the elderly. Zeitschrift für Gerontologie und Geriatrie, 33(5), 374-380.

https://link.springer.com/article/10.1007/s00391007 0034

Schwaiblmair, M., Behr, W., Haeckel, T., Märkl, B., Foerg, W., \& Berghaus, T. (2012). Drug induced interstitial lung disease. The open respiratory medicine journal, 6,63 . doi.org/10.2174/1874306401206010063

Thomas, A. Q., Lane, K., Phillips III, J., Prince, M., Markin, C., Speer, M., ... \& Loyd, J. E. (2002). Heterozygosity for a surfactant protein $\mathrm{C}$ gene mutation associated with usual interstitial pneumonitis and cellular nonspecific interstitial pneumonitis in one kindred. American journal of respiratory and critical care medicine, 165(9), 1322-1328. doi.org/10.1164/rccm.200112-123OC

Chung, M. J., Goo, J. M., \& Im, J. G. (2004). Pulmonary tuberculosis in patients with idiopathic pulmonary fibrosis. European journal of radiology, 52(2), 175-179. doi.org/10.1016/j.ejrad.2003.11.017 